\title{
Complete relations on fuzzy complete lattices ${ }^{\star \star \star}$ (Preprint)
}

\author{
Jan Konecny and Michal Krupka \\ Data Analysis and Modeling Lab, \\ Dept. of Computer Science, Palacký University, Olomouc \\ 17. listopadu 12, Olomouc, Czech Republic \\ jan.konecny@upol.cz \\ michal.krupka@upol.cz
}

\begin{abstract}
We generalize the notion of complete binary relation on complete lattice to residuated lattice valued ordered sets and show its properties. Then we focus on complete fuzzy tolerances on fuzzy complete lattices and prove they are in one-to-one correspondence with extensive isotone Galois connections. Finally, we prove that fuzzy complete lattice, factorized by a complete fuzzy tolerance, is again a fuzzy complete lattice.
\end{abstract}

\section{Introduction}

In classical algebra, a complete relation on a complete lattice is a relation which preserves arbitrary infima and suprema. For instance, a binary relation $\sim$ on a complete lattice $U$ is complete, if for each system $\left\{\left\langle u_{i}, v_{i}\right\rangle\right\}_{i \in I}$ of pairs of elements from $U, u_{i} \sim v_{i}$ for each $i \in I$ implies $\bigwedge_{i \in I} u_{i} \sim \bigwedge_{i \in I} v_{i}$ and $\bigvee_{i \in I} u_{i} \sim \bigvee_{i \in I} v_{i}$

One of the goals of this paper is to define a notion of complete relation for fuzzy sets. That is, we need to state an appropriate condition for completeness of a fuzzy relation on a set, possessing an appropriate structure of a complete lattice in fuzzy sense. However, the above definition cannot be used as is.

As it turns out, there is an equivalent condition to that of completeness of a relation on a complete lattice, that involves extending relations between sets to relations between power sets (i.e. sets of all subsets). This situation is known from the theory of so called power algebras [6], which offers a natural way to extend a binary relation $R$ on a set $X$ to a binary relation $R^{+}$on the power set $2^{X}$.

This extension allows us formulate the following equivalent condition for completeness of binary relations: a binary relation $\sim$ on a complete lattice $\mathbf{U}$ is complete, if and only if for any two subsets $V_{1}, V_{2}$ in $\mathbf{U}, V_{1} \sim^{+} V_{2}$ implies $\wedge V_{1} \sim \wedge V_{2}$ and $\bigvee V_{1} \sim \bigvee V_{2}$.

In [9], Georgescu extended the theory of power algebras to a fuzzy setting. He shows a way of extending any fuzzy $n$-ary relation $R$ on a set $X$ to a fuzzy $n$-ary relation on

\footnotetext{
* This paper is an extended and thoroughly rewritten version of a part of a paper presented at CLA 2011. We also fix some inaccuracies that appeared in the original paper.

${ }^{\star \star}$ The authors acknowledge support by the ESF project No. CZ.1.07/2.3.00/20.0059, the project is co-financed by the European Social Fund and the state budget of the Czech Republic.
} 
the set of all fuzzy sets in $X$. In this paper, we use these results to define a notion of a complete binary fuzzy relation on a complete fuzzy lattice.

As a general framework, we use $\mathbf{L}$-valued fuzzy sets, where $\mathbf{L}$ is a complete residuated lattice, thus covering $[0,1]$-valued fuzzy sets with arbitrary left-continuous t-norm on $[0,1]$ as a special case. Under this framework, we use a notion of $\mathbf{L}$-ordered set, which is, basically, a set with an L-relation satisfying requirements of reflexivity, antisymmetry and transitivity. A complete fuzzy lattice, or, more precisely, a completely lattice $\mathbf{L}$-ordered set, is then an $\mathbf{L}$-ordered set whose each $\mathbf{L}$-subset has a (properly defined) infimum and supremum.

$\mathbf{L}$-valued fuzzy sets, completely lattice $\mathbf{L}$-ordered sets and other basic notions from the fuzzy set theory (e.g. isotone $\mathbf{L}$-Galois connections and $\mathbf{L}$-closure and $\mathbf{L}$-interior operators) are introduced in Sec.2 In this section, we also prove some basic new results we need in subsequent parts of the paper, namely some properties on isotone $\mathbf{L}$-Galois connections.

Sec. 3 is devoted to some basic parts of the Georgescu's theory of fuzzy power structures and its applications to $\mathbf{L}$-ordered sets. We start with recalling the notion of power binary $\mathbf{L}$-relations and their basic properties and then we prove some results on power relations of $\mathbf{L}$-orders. Section 4 contains our definition of complete binary $\mathbf{L}$ relation on completely lattice $\mathbf{L}$-ordered set. We also prove some basic properties of complete L-relations.

In the main part of the paper, Section 5, we focus on complete fuzzy tolerances. A (crisp) tolerance on a set is a reflexive and symmetric binary relation. A block of a tolerance is a set whose elements are pairwise related. A maximal block is a block which is maximal w.r.t. set inclusion. The set of all maximal blocks of a tolerance is called the factor set. One of basic results on tolerances on complete lattices is that complete lattices can be factorized by complete tolerances [7|16]. That is, there can be introduced in a natural way an ordering on the set of all maximal blocks of a complete tolerance, such that the factor set, together with this ordering, is again a complete lattice.

We show that the same holds for complete $\mathbf{L}$-tolerances on completely lattice $\mathbf{L}$ ordered sets. More precisely, we use the usual definition of fuzzy tolerance and corresponding factor set and introduce an $\mathbf{L}$-order on the factor set of completely lattice $\mathbf{L}$-ordered set by a complete $\mathbf{L}$-tolerance, such that the new $\mathbf{L}$-order is again a complete lattice $\mathbf{L}$-order.

To prove this main result, we investigate deeply properties of complete $\mathbf{L}$-tolerances. We use similar techniques to those used in classical ordered sets. However, we also introduce a result that is new even in the classical case: we show that complete fuzzy tolerances are in one-to-one correspondence with so-called extensive isotone fuzzy Galois connections.

Note that factorization of complete lattices, either in ordinary or fuzzy setting, has been studied in the past [16/8/4/2|14] as it is useful for reducing dimensionality of concept lattices. This paper can be viewed as a contribution to this area. 


\section{Preliminaries}

\subsection{Residuated lattices and fuzzy sets}

A complete residuated lattice [2]11[15] is a structure $\mathbf{L}=\langle L, \wedge, \vee, \otimes, \rightarrow, 0,1\rangle$ such that

(i) $\langle L, \wedge, \vee, 0,1\rangle$ is a complete lattice, i.e. a partially ordered set in which arbitrary infima and suprema exist, $0=\bigwedge L, 1=\bigvee L$

(ii) $\langle L, \otimes, 1\rangle$ is a commutative monoid, i.e. $\otimes$ is a binary operation which is commutative, associative, and $a \otimes 1=a$ for each $a \in L$;

(iii) $\otimes$ and $\rightarrow$ satisfy adjointness, i.e. $a \otimes b \leq c$ iff $a \leq b \rightarrow c$.

The partial order of $\mathbf{L}$ is denoted by $\leq$. Throughout the paper, $\mathbf{L}$ denotes an arbitrary complete residuated lattice.

Elements of $L$ are called truth degrees. $\otimes$ and $\rightarrow$ are (truth functions of) "fuzzy conjunction" and "fuzzy implication".

Common examples of complete residuated lattices include those defined on $[0,1]$, (i.e. $L=[0,1]), \wedge$ being minimum, $\vee$ maximum, $\otimes$ being a left-continuous t-norm with the corresponding $\rightarrow$.

The three most important pairs of adjoint operations on the unit interval are

$$
\begin{aligned}
& \text { Lukasiewicz: } \quad \begin{aligned}
a \otimes b & =\max (a+b-1,0) \\
a \rightarrow b & =\min (1-a+b, 1)
\end{aligned} \\
& \text { Gödel: } \quad a \rightarrow b=\left\{\begin{array}{l}
1 a \leq b \\
b \text { otherwise }
\end{array}\right. \\
& a \otimes b=a \cdot b \\
& \text { Goguen (product): } a \rightarrow b= \begin{cases}1 & a \leq b \\
\frac{b}{a} \text { otherwise }\end{cases}
\end{aligned}
$$

An $\mathbf{L}$-set (or fuzzy set) $A$ in a universe set $X$ is a mapping assigning to each $x \in X$ some truth degree $A(x) \in L$. The set of all $\mathbf{L}$-sets in a universe $X$ is denoted $L^{X}$.

The operations with $\mathbf{L}$-sets are defined elementwise. For instance, the intersection of $\mathbf{L}$-sets $A, B \in L^{X}$ is an $\mathbf{L}$-set $A \cap B$ in $X$ such that $(A \cap B)(x)=A(x) \wedge B(x)$ for each $x \in X$, etc. An $\mathbf{L}$-set $A \in L^{X}$ is also denoted $\{A(x) / x \mid x \in X\}$. If for all $y \in X$ distinct from $x_{1}, x_{2}, \ldots, x_{n}$ we have $A(y)=0$, we also write $\left\{{ }^{A\left(x_{1}\right)} / x_{1},{ }^{A\left(x_{2}\right)} / x_{1}, \ldots,{ }^{A\left(x_{n}\right)} / x_{n}\right\}$.

Binary L-relations (binary fuzzy relations) between $X$ and $Y$ can be thought of as $\mathbf{L}$-sets in the universe $X \times Y$. That is, a binary $\mathbf{L}$-relation $I \in L^{X \times Y}$ between a set $X$ and a set $Y$ is a mapping assigning to each $x \in X$ and each $y \in Y$ a truth degree $I(x, y) \in L$ (a degree to which $x$ and $y$ are related by $I$ ). The inverse relation $I^{-1}$ to the $\mathbf{L}$-relation $I$ is an $\mathbf{L}$-set in $Y \times X$ and is defined by $I^{-1}(y, x)=I(x, y)$.

The composition $R \circ T$ of binary $\mathbf{L}$-relations $R \in L^{X \times Y}$ and $T \in L^{Y \times Z}$ [12] is a binary L-relation between $X$ and $Z$ defined by

$$
(R \circ T)(x, z)=\bigvee_{y \in Y} R(x, y) \otimes T(y, z) .
$$


$\mathbf{L}$-sets in a set $X$ can be naturally identified with binary $\mathbf{L}$-relations between $\{1\}$ and $X$, resp. $X$ and $\{1\}$. Thus, we can also consider composition of an $\mathbf{L}$-sets and a binary $\mathbf{L}$-relation and even composition of two $\mathbf{L}$ set: for $A, A_{1}, A_{2} \in \mathbf{L}^{X}, B \in \mathbf{L}^{Y}$ and $R \in \mathbf{L}^{X \times Y}$ we have

$$
(A \circ R)(y)=\bigvee_{x \in X} A(x) \otimes R(x, y), \quad(R \circ B)(x)=\bigvee_{y \in Y} R(x, y) \otimes B(y),
$$

and

$$
A_{1} \circ A_{2}=\bigvee_{x \in X} A_{1}(x) \otimes A_{2}(x) .
$$

An $\mathbf{L}$-set $A \in L^{X}$ is called crisp if $A(x) \in\{0,1\}$ for each $x \in X$. Crisp $\mathbf{L}$-sets can be identified with ordinary sets. For a crisp $\mathbf{L}$-set $A$, we also write $x \in A$ for $A(x)=1$ and $x \notin A$ for $A(x)=0$. An $\mathbf{L}$-set $A \in L^{X}$ is called empty (denoted by $\emptyset$ ) if $A(x)=0$ for each $x \in X$. For $a \in L$ and $A \in L^{X}, a \otimes A \in L^{X}$ and $a \rightarrow A \in L^{X}$ are defined by

$$
(a \otimes A)(x)=a \otimes A(x) \text { and }(a \rightarrow A)(x)=a \rightarrow A(x) .
$$

For an $\mathbf{L}$-set $A \in L^{X}$ and $a \in L$, the $a$-cut of $A$ is a crisp subset ${ }^{a} A \subseteq X$ such that $x \in{ }^{a} A$ iff $a \leq A(x)$. This definition applies also to binary $\mathbf{L}$-relations, whose $a$-cuts are classical (crisp) binary relations.

For a universe $X$ we define an $\mathbf{L}$-relation of graded subsethood $L^{X} \times L^{X} \rightarrow L$ by:

$$
S(A, B)=\bigwedge_{x \in X} A(x) \rightarrow B(x) .
$$

Graded subsethood generalizes the classical subsethood relation $\subseteq$; indeed, in the crisp case (i.e. $L=\{0,1\}$ ) (4) becomes $S(A, B)=1$ iff for each $x \in X: x \in A$ implies $y \in B$. Note that $S$ is a binary $\mathbf{L}$-relation on $L^{X}$. Described verbally, $S(A, B)$ represents a degree to which $A$ is a subset of $B$. In particular, we write $A \subseteq B$ iff $S(A, B)=1$. As a consequence, we have $A \subseteq B$ iff $A(x) \leq B(x)$ for each $x \in X$.

Further we set

$$
A \approx^{X} B=S(A, B) \wedge S(B, A) .
$$

The value $A \approx^{X} B$ is interpreted as the degree to which the sets $A$ and $B$ are similar.

A binary $\mathbf{L}$-relation $R$ on a set $X$ is called reflexive if $R(x, x)=1$ for any $x \in X$, symmetric if $R(x, y)=R(y, x)$ for any $x, y \in X$, and transitive if $R(x, y) \otimes R(y, z) \leq R(x, z)$ for any $x, y, z \in X . R$ is called an $\mathbf{L}$-tolerance, if it is reflexive and symmetric, $\mathbf{L}$-equivalence if it is reflexive, symmetric and transitive. If $R$ is an $\mathbf{L}$-equivalence such that for any $x, y \in X$ from $R(x, y)=1$ it follows $x=y$, then $R$ is called an $\mathbf{L}$-equality on $X$. $\mathbf{L}$ equalities are often denoted by $\approx$. The similarity $\approx^{X}$ of $\mathbf{L}$-sets $(5)$ is an $\mathbf{L}$-equality on $L^{X}$.

Let $\sim$ be an $\mathbf{L}$-equivalence on $X$. We say that an $\mathbf{L}$-set $A$ in $X$ is compatible with $\sim$ (or extensional w.r.t. $\sim$, if for any $x, x^{\prime} \in X$ it holds

$$
A(x) \otimes\left(x \sim x^{\prime}\right) \leq A\left(x^{\prime}\right) .
$$


A binary $\mathbf{L}$-relation $R$ on $X$ is compatible with $\sim$, if for each $x, x^{\prime}, y, y^{\prime} \in X$,

$$
R(x, y) \otimes\left(x \sim x^{\prime}\right) \otimes\left(y \sim y^{\prime}\right) \leq R\left(x^{\prime}, y^{\prime}\right) .
$$

Zadeh's extension principle [17] allows extending any mapping $f: X \rightarrow Y$ to a mapping $f^{+}: L^{X} \rightarrow L^{Y}$ by setting for each $A \in L^{X}$

$$
f^{+}(A)(y)=\bigvee_{x \in X, f(x)=y} A(x) .
$$

In the following we use well-known properties of residuated lattices and fuzzy structures which can be found e.g. in [2]11].

\subsection{L-ordered sets}

In this section, we recall basic definitions and results of the theory of $\mathbf{L}$-ordered sets. Basic references are [1]2] and the references therein.

An $\mathbf{L}$-order on a set $U$ with an $\mathbf{L}$-equality $\approx$ is a binary $\mathbf{L}$-relation $\preceq$ on $U$ which is compatible with $\approx$, reflexive, transitive and satisfies $(u \preceq v) \wedge(v \preceq u) \leq u \approx v$ for any $u, v \in U$ (antisymmetry). The tuple $\mathbf{U}=\langle\langle U, \approx\rangle, \preceq\rangle$ is called an $\mathbf{L}$-ordered set. An immediate consequence of the definition is that for any $u, v \in U$ it holds

$$
u \approx v=(u \preceq v) \wedge(v \preceq u) .
$$

If $\mathbf{U}=\langle\langle U, \approx\rangle, \preceq\rangle$ is an $\mathbf{L}$-ordered set, then the tuple $\left\langle U,{ }^{1} \preceq\right\rangle$, where ${ }^{1} \preceq$ is the 1cut of $\preceq$, is a (partially) ordered set. We sometimes write $\leq$ instead of ${ }^{1} \preceq$ and use the symbols $\wedge, \wedge$ resp. $\vee, \bigvee$ for denoting infima resp. suprema in $\left\langle U,{ }^{1} \preceq\right\rangle$.

For two $\mathbf{L}$-ordered sets $\mathbf{U}=\left\langle\left\langle U, \approx_{U}\right\rangle, \preceq_{U}\right\rangle$ and $\mathbf{V}=\left\langle\left\langle V, \approx_{V}\right\rangle, \preceq_{V}\right\rangle$, a mapping $f$ : $U \rightarrow V$ is isotone, if $\left(u_{1} \preceq_{U} u_{2}\right) \leq\left(f\left(u_{1}\right) \preceq_{V} f\left(u_{2}\right)\right)$ for any $u_{1}, u_{2} \in V$. The mapping $f$ is called an isomorphism of $\mathbf{U}$ and $\mathbf{V}$, if it is a bijection and $\left(u_{1} \preceq_{U} u_{2}\right)=\left(f\left(u_{1}\right) \preceq_{V}\right.$ $\left.f\left(u_{2}\right)\right)$ for any $u_{1}, u_{2} \in V$. $\mathbf{U}$ and $\mathbf{V}$ are then called isomorphic.

In classical theory of ordered sets, a subset $V$ of an ordered set is called a lower set, if for each element $u$ such that there is $v \in V$ satisfying $u \leq v$, it holds $u \in V$. Equivalently, for a lower set $V$ it holds: if $u \leq v$, then $v \in V$ implies $u \in V$.

Analogously, for an $\mathbf{L}$-ordered set $\mathbf{U}$, an $\mathbf{L}$-set $V \in L^{U}$ is called a lower set (resp. an upper set), if for each $u, v \in U$ it holds

$$
u \preceq v \leq V(v) \rightarrow V(u) \quad(\text { resp. } u \preceq v \leq V(u) \rightarrow V(v)) .
$$

The lower (resp. upper) set of an $\mathbf{L}$-set $V \in L^{U}$ is the $\mathbf{L}$-set $\downarrow V$ (resp. $\uparrow V$ ), defined by

$$
\begin{aligned}
& \downarrow V(u)=(\preceq \circ V)(u)=\bigvee_{v \in U}(u \preceq v) \otimes V(v), \\
& \uparrow V(u)=(V \circ \preceq)(u)=\bigvee_{v \in U}(v \preceq u) \otimes V(v) .
\end{aligned}
$$

In a similar manner we define lower and upper cone of $V \in L^{U}$. For any $v \in U$ we set

$$
\mathscr{L} V(v)=\bigwedge_{u \in U} V(u) \rightarrow(v \preceq u), \quad \mathscr{U} V(v)=\bigwedge_{u \in U} V(u) \rightarrow(u \preceq v) .
$$


The right-hand side of the first equation is the degree of "For each $u \in U$, if $u$ is in $V$, then $v$ is less than or equal to $u$ ", and similarly for the second equation. Thus, $\mathscr{L} V(v)$ $(\mathscr{U} V(v))$ can be seen as the degree to which $v$ is less (greater) than or equal to each element of $V$, that is the degree to which $v$ is a lower (upper) bound of $V$.

In the case $\mathscr{L} V(v)=1$ (resp. $\mathscr{U} V(v)=1$ ) we say simply $v$ is a lower (upper) bound of $V . \mathscr{L} V$ (resp. $\mathscr{U} V$ ) is called the $\mathbf{L}$-set of lower bounds (resp. upper bounds) of $V$, or the lower cone (resp. the upper cone) of $V$.

If $u, v \in U, v \leq u$, then the $\mathbf{L}$-set $\llbracket v, u \rrbracket=\mathscr{U}\{v\} \cap \mathscr{L}\{u\}$ is called an $\mathbf{L}$-interval (or simply an interval) in $\mathbf{U}$.

We set $[v, u]={ }^{1} \llbracket v, u \rrbracket$. Thus, $[v, u]$ denotes the classical interval with respect to the 1-cut of $\preceq:[v, u]=\left\{u^{\prime} \mid v \leq u^{\prime} \leq u\right\}$.

An $\mathbf{L}$-set $V \in L^{U}$ is convex if $V=\downarrow V \cap \uparrow V$. The " $\subseteq$ " inclusion always holds as the lower set as well as upper set of $V$ always contain $V$ as a subset. For each $V \in L^{U}$, each of the following $\mathbf{L}$-sets is convex: $\downarrow V, \uparrow V, \mathscr{L} V, \mathscr{U} V$. Every $\mathbf{L}$-interval $\llbracket v, u \rrbracket$ in $\mathbf{U}$ is also convex. Every convex $\mathbf{L}$-set in $\mathbf{U}$ is compatible with $\approx$.

In the following two lemmas we formulate basic properties of lower and upper sets and cones that will be needed in the sequel. All the properties can be proved by direct computation.

Lemma 1. For each $V \in L^{U}$ we have

$$
\begin{aligned}
\downarrow V & =\downarrow \downarrow V, & \uparrow V & =\uparrow \uparrow V, \\
\mathscr{L} V & =\downarrow \mathscr{L} V=\mathscr{L} \uparrow V, & \mathscr{U} V & =\uparrow \mathscr{U} V=\mathscr{U} \downarrow V .
\end{aligned}
$$

Lemma 2. For each $V, V_{1}, V_{2} \in L^{U}, u, v \in U$ we have

$$
\begin{array}{rlrl}
S\left(V_{1}, V_{2}\right) & \leq S\left(\mathscr{L} V_{2}, \mathscr{L} V_{1}\right), & S\left(V_{1}, V_{2}\right) & \leq S\left(\mathscr{U} V_{2}, \mathscr{U} V_{1}\right), \\
\mathscr{L} \mathscr{U} \mathscr{L} V & =\mathscr{L} V, & \mathscr{U} \mathscr{L} \mathscr{U} V & =\mathscr{U} V \\
V & \subseteq \mathscr{U} \mathscr{L} V & V & \subseteq \mathscr{L} \mathscr{U} V \\
\mathscr{L}\left(V_{1} \cup V_{2}\right) & =\mathscr{L} V_{1} \cap \mathscr{L} V_{2}, & \mathscr{U}\left(V_{1} \cup V_{2}\right) & =\mathscr{U} V_{1} \cap \mathscr{U} V_{2} \\
\mathscr{L}\{v\}(u) & =u \preceq v, & \mathscr{U}\{v\}(u) & =v \preceq u, \\
\mathscr{L} \mathscr{U}\{v\} & =\mathscr{L}\{v\}, & \mathscr{U} \mathscr{L}\{v\} & =\mathscr{U}\{v\}, \\
u \preceq v & =S(\mathscr{L}\{u\}, \mathscr{L}\{v\}), & u \preceq v & =S(\mathscr{U}\{v\}, \mathscr{U}\{u\}) .
\end{array}
$$

\subsection{Completely lattice L-ordered sets}

For any $\mathbf{L}$-set $V \in L^{U}$ there exists at most one element $u \in U$ such that $\mathscr{L} V(u) \wedge$ $\mathscr{U}(\mathscr{L} V)(u)=1$ (resp. $\mathscr{U} V \wedge \mathscr{L}(\mathscr{U} V)(u)=1)$ [112]. If there is such an element, we call it the infimum of $V$ (resp. the supremum of $V$ ) and denote $\inf V($ resp. $\sup V)$; otherwise we say that the infimum (resp. supremum) does not exist.

If $\inf V$ exists and $V(\inf V)=1$, then it is called minimum of $V$ and denoted $\min V$. Similarly, if $\sup V$ exists and $V(\sup V)=1$, then we call it maximum of $V$ and denote $\max V$.

Infimum (supremum) of $V$ is obviously a lower (upper) bound of $V$ and, in the same time, an upper bound of $\mathscr{L} V$ (a lower bound of $\mathscr{U} V$ ). 
Lemma 3. If $\inf V$ exists, then $\mathscr{L} V=\mathscr{L}\{\inf V\}$. If $\sup V$ exists, then $\mathscr{U} V=\mathscr{U}\{\sup V\}$.

Proof. By definition, $\mathscr{L} V \supseteq\{\inf V\}$. Applying both inequalities from (16) we obtain $\mathscr{L} \mathscr{U} \mathscr{L} V \supseteq \mathscr{L} \mathscr{U}\{\inf V\}$. By (17), $\mathscr{L} \mathscr{U} \mathscr{L} V=\mathscr{L} V$ and by (21), $\mathscr{L} \mathscr{U}\{\inf V\}=$ $\mathscr{L}\{\inf V\}$. Thus, $\mathscr{L} V \supseteq \mathscr{L}\{\inf V\}$.

By definition of lower cone again, $\{\inf V\} \subseteq \mathscr{U} \mathscr{L} V$. The first inequality of (16) gives $\mathscr{L} \mathscr{U} \mathscr{L} V \subseteq \mathscr{L}\{\inf V\}$ and by [17], $\mathscr{L} V \subseteq \mathscr{L}\{\inf V\}$.

The proof for upper cones is similar.

Lemma 4. If $\inf V$ exists, then $V \subseteq \mathscr{U}\{\inf V\}$. If $\sup V$ exists, then $V \subseteq \mathscr{L}\{\sup V\}$.

Proof. By (18), Lemma 3, (21), $V \subseteq \mathscr{U} \mathscr{L} V=\mathscr{U} \mathscr{L}\{\inf V\}=\mathscr{U}\{\inf V\}$. The second part is dual.

An $\mathbf{L}$-ordered set $\mathbf{U}$ is called completely lattice $\mathbf{L}$-ordered, if for each $V \in L^{U}$, both $\inf V$ and $\sup V$ exist.

An important example of a completely lattice $\mathbf{L}$-ordered set is the following. For a set $X$, the tuple $\left\langle\left\langle L^{X}, \approx^{X}\right\rangle, S\right\rangle$ is a completely lattice $\mathbf{L}$-ordered set with infima and suprema given by

$$
(\inf V)(u)=\bigwedge_{W \in L^{X}} V(W) \rightarrow W(u), \quad(\sup V)(u)=\bigvee_{W \in L^{X}} V(W) \otimes W(u) .
$$

This fact follows easily e.g. from the main theorem of fuzzy concept lattices (fuzzy order version) [2]1].

Note that from definition and 177 it follows

$$
\inf V=\max \mathscr{L} V, \quad \sup V=\max \mathscr{U} V .
$$

Thus, to show $\mathbf{U}$ is a completely lattice $\mathbf{L}$-ordered set it suffices to prove existence of suprema resp. infima of all $\mathbf{L}$-sets in $U$.

Consequently, the following holds for infima and suprema of $\mathbf{L}$-intervals:

$$
v=\min \llbracket v, u \rrbracket, \quad u=\max \llbracket v, u \rrbracket .
$$

Lemma 5. The following holds for any $\mathbf{L}$-sets $V_{1}, V_{2}$ in a completely lattice $\mathbf{L}$-ordered set $\mathbf{U}$.

$$
\begin{aligned}
S\left(\mathscr{L} V_{1}, \mathscr{L} V_{2}\right) & =\inf V_{1} \preceq \inf V_{2}, & S\left(\mathscr{U} V_{1}, \mathscr{U} V_{2}\right) & =\sup V_{2} \preceq \sup V_{1}, \\
S\left(V_{1}, V_{2}\right) & \leq \inf V_{2} \preceq \inf V_{1}, & S\left(V_{1}, V_{2}\right) & \leq \sup V_{1} \preceq \sup V_{2}
\end{aligned}
$$

Proof. By Lemma 3 and (22), $S\left(\mathscr{L} V_{1}, \mathscr{L} V_{2}\right)=S\left(\mathscr{L}\left\{\inf V_{1}\right\}, \mathscr{L}\left\{\inf V_{2}\right\}\right)=\inf V_{1} \preceq$ $\inf V_{2}$, proving the first part of (26). The second part is dual. (27) follows from (26) by (16).

Lemma 6. The following holds for each $u, v \in U$ :

$$
\inf \{v \preceq u / u, v\}=v, \quad \sup \{u \preceq v / u, v\}=v .
$$

Proof. By direct computation. 


\subsection{Isotone mappings of L-ordered sets}

We prove some basic properties of isotone mappings of $\mathbf{L}$-ordered sets we will need later. The following lemma says that isotone mappings transform lower (upper) bounds of an $\mathbf{L}$-set to lower (upper) bounds of its image.

Lemma 7. Let $f: U \rightarrow U^{\prime}$ be an isotone mapping of $\mathbf{L}$-ordered sets, $V \in L^{U}$. Then

$$
f(\mathscr{L} V) \subseteq \mathscr{L} f(V), \quad f(\mathscr{U} V) \subseteq \mathscr{U} f(V) .
$$

Proof. By definition of lower cone and isotony of $f$,

$$
\mathscr{L} V(v) \leq V(u) \rightarrow(v \preceq u) \leq V(u) \rightarrow(f(v) \preceq f(u)),
$$

for each $u, v \in U$. Now let $u^{\prime} \in U^{\prime}$ and take infimum for all $u$ such that $f(u)=u^{\prime}$ (in the case there is no such $u$, the infimum, as the infimum of empty set in $L$, is equal to $1 \in L)$ :

$$
\begin{aligned}
\mathscr{L} V(v) & \leq \bigwedge_{f(u)=u^{\prime}} V(u) \rightarrow(f(v) \preceq f(u))=\left(\bigvee_{f(u)=u^{\prime}} V(u)\right) \rightarrow(f(v) \preceq f(u)) \\
& =f(V)\left(u^{\prime}\right) \rightarrow\left(f(v) \preceq u^{\prime}\right) .
\end{aligned}
$$

Now denote $v^{\prime}=f(v)$. The above inequality tells that the following holds for each $v \in U$ such that $f(v)=v^{\prime}$ and $u^{\prime} \in U^{\prime}$ :

$$
\mathscr{L} V(v) \leq f(V)\left(u^{\prime}\right) \rightarrow\left(v^{\prime} \preceq u^{\prime}\right) .
$$

Thus,

$$
f(\mathscr{L} V)\left(v^{\prime}\right)=\bigvee_{f(v)=v^{\prime}} \mathscr{L} V(v) \leq \bigwedge_{u^{\prime} \in U^{\prime}} f(V)\left(u^{\prime}\right) \rightarrow\left(v^{\prime} \preceq u^{\prime}\right)=\mathscr{L} f(V)\left(v^{\prime}\right) .
$$

This proves the first inclusion, the second one is similar.

Let $v \in U$. As it has been said, $\mathscr{L} V(v)$ is the degree to which $v$ is a lower bound of $V$. We have $\mathscr{L} V(v) \leq f(\mathscr{L} V)(f(v))$ and by the above lemma, $f(\mathscr{L} V)(f(v)) \leq$ $\mathscr{L} f(V)(f(v))$. This way the lemma tells that the degree to which $f(v)$ is a lower bound of $f(V)$ is greater than or equal to the degree to which $v$ is a lower bound of $V$ (and similarly for upper bounds). In the particular case $\mathscr{L} V(v)=1$ (or $\mathscr{U} V(v)=1$ ) we obtain the following result:

Corollary 1. In the setting of the previous lemma, if $\mathscr{L} V(v)=1$, then $\mathscr{L} f(V)(f(v))=$ 1 and if $\mathscr{U} V(v)=1$, then $\mathscr{U} f(V)(f(v))=1$. In words, if $v$ is a lower (upper) bound of $V$, then $f(v)$ is a lower (upper) bound of $f(V)$.

Lemma 8. Let $f, g: U \rightarrow U$ be two mappings such that for each $u \in U, f(u) \leq u$ and $g(u) \geq u$. Then for each $V \in L^{U}$,

$$
\begin{aligned}
\mathscr{L} f(V) & \subseteq \mathscr{L} V, & \mathscr{U} f(V) \supseteq \mathscr{U} V, \\
\mathscr{L} g(V) \supseteq \mathscr{L} V, & & \mathscr{U} g(V) \subseteq \mathscr{U} V .
\end{aligned}
$$


Proof. We will prove the first inclusion only, the others being analogous. Let $v \in U$. From transitivity of $\preceq$ we have $\left(v \preceq f\left(u^{\prime}\right)\right) \leq\left(v \preceq u^{\prime}\right)$ for each $u^{\prime} \in U$. Now,

$$
\begin{aligned}
\mathscr{L} f(V)(v) & =\bigwedge_{u \in U} f(V)(u) \rightarrow(v \preceq u)=\bigwedge_{u \in U}\left(\bigvee_{f\left(u^{\prime}\right)=u} V\left(u^{\prime}\right)\right) \rightarrow(v \preceq u) \\
& =\bigwedge_{u \in U} \bigwedge_{f\left(u^{\prime}\right)=u} V\left(u^{\prime}\right) \rightarrow(v \preceq u)=\bigwedge_{u^{\prime} \in U} V\left(u^{\prime}\right) \rightarrow\left(v \preceq f\left(u^{\prime}\right)\right) \\
& \leq \bigwedge_{u^{\prime} \in U} V\left(u^{\prime}\right) \rightarrow\left(v \preceq u^{\prime}\right)=\mathscr{L} V(v),
\end{aligned}
$$

proving the inclusion.

In the last two lemmas we suppose $\mathbf{U}$ and $\mathbf{U}^{\prime}$ are completely lattice $\mathbf{L}$-ordered sets.

Lemma 9. Let $f, g$ be the same as in the previous lemma, $V \in L^{U}$. Then

$$
\begin{array}{ll}
\inf f(V) \leq \inf V, & \sup f(V) \leq \sup V, \\
\inf g(V) \geq \inf V, & \sup g(V) \geq \sup V .
\end{array}
$$

Proof. Follows from Lemma 8 and (26).

Lemma 10. Let $f: U \rightarrow U^{\prime}$ be an isotone mapping, $V \in L^{U}$. Then

$$
f(\inf V) \leq \inf f(V), \quad f(\sup V) \geq \sup f(V) .
$$

Proof. Follows directly from Corollary 1 (e.g., inf $V$ is a lower bound of $V$, whence $f(\inf V)$ is a lower bound of $f(V)$ and hence is less than or equal to $\inf f(V)$ ).

\subsection{Isotone L-Galois connections}

An isotone $\mathbf{L}$-Galois connection between $\mathbf{L}$-ordered sets $\mathbf{U}$ and $\mathbf{V}[10[13]$ is a pair $\langle f, g\rangle$, where $f: U \rightarrow V, g: V \rightarrow U$ are mappings such that for each $u \in U, v \in V$ it holds

$$
f(u) \preceq v=u \preceq g(v) .
$$

An isotone Galois connection between $\mathbf{U}$ and $\mathbf{U}$ is called simply an isotone Galois connection on $\mathbf{U}$.

By isotone $\mathbf{L}$-Galois connection between sets $X$ and $Y$ we understand an isotone $\mathbf{L}$-Galois connection between completely lattice $\mathbf{L}$-ordered sets $\mathbf{L}^{X}$ and $\mathbf{L}^{Y}$ (23).

Note that in [10] and [13], only isotone $\mathbf{L}-$ Galois connection between sets are considered. Thus, our approach is more general, but all results from [10]13] can be transferred more or less mechanically to our setting. This is also the case in Section 2.6.

Theorem 1 (basic properties of isotone $\mathbf{L}$-Galois connections). Let $\langle f, g\rangle$ be an isotone $\mathbf{L}$-Galois connection between $\mathbf{L}$-ordered sets $\mathbf{U}$ and $\mathbf{V}$. Then

(a) $u \leq g(f(u))$ for each $u \in U, f(g(v)) \leq v$ for each $v \in V$.

(b) $f$ and $g$ are isotone. 
(c) $f(g(f(u)))=f(u), g(f(g(v)))=g(v)$.

(d) Let $\mathbf{U}$ and $\mathbf{V}$ be completely lattice $\mathbf{L}$-ordered sets. For $U^{\prime} \in L^{U}$ and $V^{\prime} \in L^{V}$ we have

$$
\begin{aligned}
f\left(\inf U^{\prime}\right) & \leq \inf f\left(U^{\prime}\right), & & f\left(\sup U^{\prime}\right)=\sup f\left(U^{\prime}\right), \\
g\left(\inf V^{\prime}\right) & =\inf g\left(V^{\prime}\right), & g\left(\sup V^{\prime}\right) & \geq \sup g\left(V^{\prime}\right) .
\end{aligned}
$$

Proof. (a) By (33), from $f(u) \leq f(u)$ it follows $u \leq g(f(u))$ and from $g(v) \leq g(v)$ it follows $f(g(v)) \leq v$.

(b) By (a), $u_{2} \leq g\left(f\left(u_{2}\right)\right)$. Thus, by transitivity, $\left(u_{1} \preceq u_{2}\right)=\left(u_{1} \preceq u_{2}\right) \otimes 1=\left(u_{1} \preceq\right.$ $\left.u_{2}\right) \otimes\left(u_{2} \preceq g\left(f\left(u_{2}\right)\right)\right) \leq\left(u_{1} \preceq g\left(f\left(u_{2}\right)\right)\right)=\left(f\left(u_{1}\right) \preceq f\left(u_{2}\right)\right)$. Similarly for $g$.

(c) By (a), $f(g(f(u))) \leq f(u)$. The opposite inequality is proved by (b) and (a): $1=u \preceq g(f(u)) \leq f(u) \preceq f(g(f(u)))$. Similarly the second equality.

(d) The inequalities $f\left(\inf U^{\prime}\right) \leq \inf f\left(U^{\prime}\right), f\left(\sup U^{\prime}\right) \geq \sup f\left(U^{\prime}\right), g\left(\inf V^{\prime}\right) \leq \inf g\left(V^{\prime}\right)$, $g\left(\sup V^{\prime}\right) \geq \sup g\left(V^{\prime}\right)$ follow from (b) and Lemma 10 By (a), the fourth inequality of Lemma 9 , and the inequality $g\left(\sup V^{\prime}\right) \geq \sup g\left(V^{\prime}\right)$ we have already proved,

$$
\sup U^{\prime} \leq \sup g\left(f\left(U^{\prime}\right)\right) \leq g\left(\sup f\left(U^{\prime}\right)\right) .
$$

Now by definition, $f\left(\sup U^{\prime}\right) \leq \sup f\left(U^{\prime}\right)$. The remaining inequality, namely $g\left(\inf V^{\prime}\right) \geq$ $\inf g\left(V^{\prime}\right)$, is proved similarly.

Let $\langle f, g\rangle$ be an isotone $\mathbf{L}$-Galois connection between $\mathbf{U}$ and $\mathbf{V}$. A pair $\langle u, v\rangle$, where $u \in U$ and $v \in V$, is called a fixpoint of $\langle f, g\rangle$ if $f(u)=v$ and $g(v)=u$.

Suppose $\left\langle u_{1}, v_{1}\right\rangle,\left\langle u_{2}, v_{2}\right\rangle$ are two fixpoints of $\langle f, g\rangle$. We have by (33),

$$
u_{1} \preceq u_{2}=u_{1} \preceq g\left(v_{2}\right)=f\left(u_{1}\right) \preceq v_{2}=v_{1} \preceq v_{2}
$$

and by (9),

$$
u_{1} \approx u_{2}=v_{1} \approx v_{2} \text {. }
$$

We denote the set of all fixpoints of $\langle f, g\rangle$ by $\operatorname{Fix}_{\langle f, g\rangle}$. For L-relations $\approx_{\operatorname{Fix}_{\langle f, g\rangle}}$ and $\preceq_{\mathrm{Fix}_{\langle f, g\rangle}}$ defined on $\operatorname{Fix}_{\langle f, g\rangle}$ by

$$
\begin{array}{ll}
\left\langle u_{1}, v_{1}\right\rangle \approx_{\mathrm{Fix}_{\langle f, g\rangle}}\left\langle u_{2}, v_{2}\right\rangle=u_{1} \approx u_{2} & \left(=v_{1} \approx v_{2}\right), \\
\left\langle u_{1}, v_{1}\right\rangle \preceq \mathrm{Fix}_{\langle f, g\rangle}\left\langle u_{2}, v_{2}\right\rangle=u_{1} \preceq u_{2} \quad\left(=v_{1} \preceq v_{2}\right),
\end{array}
$$

we obtain an $\mathbf{L}$-ordered set $\left.\operatorname{Fix}_{\langle f, g\rangle}=\left\langle\left\langle\operatorname{Fix}_{\langle f, g\rangle}\right\rangle \approx_{\left.\operatorname{Fix}_{\langle f, g\rangle}\right\rangle}\right\rangle, \preceq_{\left.\operatorname{Fix}_{\langle f, g\rangle}\right\rangle}\right\rangle$. In the rest of the paper, we will usually write $\approx$ instead of $\approx_{\mathrm{Fix}_{\langle f, g\rangle}}$ and $\preceq_{\text {instead of }} \preceq_{\left.\mathrm{Fix}_{\langle f, g}\right\rangle}$.

We denote the set of all isotone Galois connections between $\mathbf{L}$-ordered sets $\mathbf{U}$ and $\mathbf{V}$ by $\operatorname{IGal}(\mathbf{U}, \mathbf{V})$ and consider the following binary $\mathbf{L}$-relations $\approx_{\operatorname{IGal}(\mathbf{U}, \mathbf{V})}, \preceq_{\mathrm{IGal}(\mathbf{U}, \mathbf{V})}$ on $\operatorname{IGal}(\mathbf{U}, \mathbf{V})$ :

$$
\begin{aligned}
& \left\langle f_{1}, g_{1}\right\rangle \approx_{\mathrm{IGal}(\mathbf{U}, \mathbf{V})}\left\langle f_{2}, g_{2}\right\rangle=\bigwedge_{u \in U}\left(f_{2}(u) \approx f_{1}(u)\right) \wedge \bigwedge_{v \in V}\left(g_{1}(v) \approx g_{2}(v)\right), \\
& \left\langle f_{1}, g_{1}\right\rangle \preceq_{\mathrm{IGal}(\mathbf{U}, \mathbf{V})}\left\langle f_{2}, g_{2}\right\rangle=\bigwedge_{u \in U}\left(f_{2}(u) \preceq f_{1}(u)\right) \wedge \bigwedge_{v \in V}\left(g_{1}(v) \preceq g_{2}(v)\right) .
\end{aligned}
$$


Lemma 11. $\left\langle\left\langle\operatorname{IGal}(\mathbf{U}, \mathbf{V}), \approx_{\mathrm{IGal}(\mathbf{U}, \mathbf{V})}\right\rangle, \preceq_{\mathrm{IGal}(\mathbf{U}, \mathbf{V})}\right\rangle$ is an $\mathbf{L}$-ordered set.

Proof. Straightforward.

For an $\mathbf{L}$-ordered set $\mathbf{U}$, an isotone $\mathbf{L}$-Galois connection $\langle f, g\rangle$ on $\mathbf{U}$ is called extensive if

$$
f(u) \leq u \quad \text { and } \quad g(u) \geq u
$$

for each $u \in U$. The set of all extensive isotone $\mathbf{L}$-Galois connections on $\mathbf{U}$ is denoted $\operatorname{EIGal}(\mathbf{U})$.

Notice that if one of the conditions (38) holds true, then the second one follows by (33).

\subsection{L-closure and L-interior operators}

Here we recall very briefly basic definitions and results on $\mathbf{L}$-closure and $\mathbf{L}$-interior operators. More details can be found in [3]1[10].

For an $\mathbf{L}$-ordered set $\mathbf{U}$, a mapping $C: U \rightarrow U$ is called an $\mathbf{L}$-closure operator, if the following holds for each $u, u_{1}, u_{2} \in U$ :

$$
\begin{aligned}
C(u) & \geq u, \\
C(C(u)) & =C(u), \\
u_{1} \preceq u_{2} & \leq C\left(u_{1}\right) \preceq C\left(u_{2}\right) .
\end{aligned}
$$

A mapping $I: U \rightarrow U$ is called an $\mathbf{L}$-interior operator, if for each $u, u_{1}, u_{2} \in U$,

$$
\begin{aligned}
I(u) & \leq u, \\
I(I(u)) & =I(u), \\
u_{1} \preceq u_{2} & \leq I\left(u_{1}\right) \preceq I\left(u_{2}\right) .
\end{aligned}
$$

By $\mathbf{L}$-closure (resp. $\mathbf{L}$-interior) operator on a set $X$ we mean an $\mathbf{L}$-closure (resp. $\mathbf{L}$ interior) operator on the completely lattice $\mathbf{L}$-ordered $\operatorname{set} \mathbf{L}^{X}(23)$.

An element $u \in U$ is a fixpoint of $C$ (resp. fixpoint of $I$ ), if $C(u)=u($ resp. $I(u)=u$ ). The set of all fixpoints of $C$ (resp. $I$ ) will be denoted Fix ${ }_{C}\left(\right.$ resp. Fix ${ }_{I}$ ). The sets Fix and $\mathrm{Fix}_{I}$ inherit a structure of an $\mathbf{L}$-ordered set from $\mathbf{U}$. Considering Fix $_{C}$ and Fix $_{I}$ with this structure we have the following result:

Theorem 2. Let $\mathbf{U}$ be a completely lattice $\mathbf{L}$-ordered set. Then Fix $_{C}$ is closed w.r.t. arbitrary infima (i.e. for any $\mathbf{L}$-set $V \in L^{U}, V \subseteq \mathrm{Fix}_{C}$, we have $\inf V \in \mathrm{Fix}_{C}$ ) and $\mathrm{Fix}_{I}$ is closed w.r.t. arbitrary suprema (i.e. for any $\mathbf{L}$-set $V \in L^{U}, V \subseteq \operatorname{Fix}_{I}$, we have $\sup V \in$ $\left.\mathrm{Fix}_{I}\right)$. Consequently, $\mathrm{Fix}_{C}$ and $\mathrm{Fix}_{I}$ are completely lattice $\mathbf{L}$-ordered sets.

A subset $V \subseteq U$ which is closed w.r.t. arbitrary infima (resp. suprema) is called an $\mathbf{L}$-closure (resp. L-interior) system in $\mathbf{U}$. The above theorem says that Fix ${ }_{C}($ resp. Fix $I$ ) is an $\mathbf{L}$-closure (resp. $\mathbf{L}$-interior) system in $\mathbf{U}$. In the case $\mathbf{U}=\mathbf{L}^{X}$ for some set $X$ we also talk about $\mathbf{L}$-closure (resp. L-interior) system in $X$. 
Let $\langle f, g\rangle$ be an isotone $\mathbf{L}$-Galois connection on $\mathbf{U}$. From Theorem 1 it easily follows that the composition $C$, given by $C(u)=g(f(u))$ is an $\mathbf{L}$-closure operator on $U$ and the composition $I, I(v)=f(g(v))$ is an $\mathbf{L}$-interior operator on $V$.

We have the following result for the $\mathbf{L}$-ordered sets of fixpoints of these operators and of the $\mathbf{L}$-Galois connection $\langle f, g\rangle$ itself:

Theorem 3. Let $\mathbf{U}$ be a completely lattice $\mathbf{L}$-ordered set. Then the $\mathbf{L}$-ordered sets $\operatorname{Fix}_{\langle f, g\rangle}, \mathrm{Fix}_{C}, \mathrm{Fix}_{I}$ are isomorphic. Consequently, $\mathrm{Fix}_{\langle f, g\rangle}$ is a completely lattice $\mathbf{L}$ ordered set. The isomorphism Fix $_{\langle f, g\rangle} \rightarrow$ Fix $_{C}$ is given by $\langle u, v\rangle \rightarrow u$ and the isomorphism $\operatorname{Fix}_{\langle f, g\rangle} \rightarrow$ Fix $_{I}$ is given by $\langle u, v\rangle \rightarrow v$.

\section{Power structures of L-ordered sets}

Power structure [6] is an algebraic structure constructed by "lifting" operations and relations on a (ordinary) set to its power set, i.e. the set of all its (ordinary) subsets. The theory goes back to Frobenius and recently [9] has been generalized to a fuzzy setting.

In this section, we recall basic definitions and results from [9] to the extent we need in this paper. We also show some results from [2]5] on fuzzy power structures. Then we prove some properties of power structures of fuzzy ordered sets we will need for the main result of this paper.

Note that in [9], fuzzy power structures are studied under the framework of continuous t-norms; generalizing results we use in this paper to complete residuated lattices is straightforward.

Let $R$ be a binary $\mathbf{L}$-relation on a set $X$. We set for any $\mathbf{L}$-sets $A, B \in L^{X}$

$$
\begin{aligned}
R^{\rightarrow}(A, B) & =S(A, R \circ B)=\bigwedge_{x \in X}\left(A(x) \rightarrow \bigvee_{y \in X} R(x, y) \otimes B(y)\right) \\
R^{\leftarrow}(A, B) & =\left(R^{-1}\right) \rightarrow(B, A)=S\left(B, R^{-1} \circ A\right)=S(B, A \circ R) \\
& =\bigwedge_{y \in X}\left(B(y) \rightarrow \bigvee_{x \in X} R(x, y) \otimes A(x)\right)
\end{aligned}
$$

Since $S(A, R \circ B)$ is the degree to which $A$ is a subset of $R \circ B, R^{\rightarrow}(A, B)$ can be viewed as the degree to which each element of $A$ is related to an element of $B$. We set

$$
R^{+}(A, B)=R^{\rightarrow}(A, B) \wedge R^{\leftarrow}(A, B),
$$

obtaining a binary $\mathbf{L}$-relation, called power $\mathbf{L}$-relation, $R^{+}$on the set $L^{X}$. In the following, we prove some basic properties of the power $\mathbf{L}$-relation $R^{+}$for $R$ being a binary $\mathbf{L}$-relation on a set $X$ and later on an $\mathbf{L}$-ordered set $\langle\langle U, \approx\rangle, \preceq\rangle$.

The following result is straightforward and has been proved in [2, Theorem 4.41].

Lemma 12. For any binary $\mathbf{L}$-relation $R \in L^{X}$ it holds

1. if $R$ is reflexive, then so is $R^{+}$,

2. if $R$ is symmetric, then so is $R^{+}$, 
3. if $R$ is transitive, then so is $R^{+}$.

The following has been proved in [5, Theorem 2].

Theorem 4. For any two L-relations $R, Q \in L^{X}$ it holds

$$
R^{+} \circ Q^{+} \subseteq(R \circ Q)^{+} .
$$

In the next two theorems we show some basic properties of power relations of $\mathbf{L}$ equivalences. We start with a lemma. Note that the $\mathbf{L}$-relation $\approx^{X}$ on $\mathbf{L}$-sets in $X$ (5) does not depend on $\sim$.

Lemma 13. Let $\sim$ be an $\mathbf{L}$-equivalence on a set $X, A, B \in L^{X}$ be compatible with $\sim$ Then $A \sim^{+} B=A \approx^{X} B$.

Proof. By compatibility, $\bigvee_{x^{\prime} \in X}\left(x \sim x^{\prime}\right) \otimes A\left(x^{\prime}\right) \leq A(x)$. As $(x \sim x) \otimes A(x)=A(x)$ the opposite inequality also holds and we have $\sim \circ A=A$. Similarly, $\sim \circ B=B$. Thus,

$$
\begin{aligned}
A \sim^{+} B & =\left(A \sim^{\rightarrow} B\right) \wedge\left(A \sim^{\leftarrow} B\right)=S(A, \sim \circ B) \wedge S(B, \sim \circ A)=S(A, B) \wedge S(B, A) \\
& =A \approx^{X} B .
\end{aligned}
$$

Theorem 5. Let $\sim$ be an $\mathbf{L}$-equality on a set $X, M \subseteq L^{X}$ a subset, containing only $\mathbf{L}$ sets, compatible with $\sim$. Then the restriction of $\sim^{+}$to $M$ is an $\mathbf{L}$-equality on $M$ and is equal to the restriction of the $\mathbf{L}$-relation $\approx^{X}(5)$.

Proof. Follows from Lemma 13 as $A \approx^{X} B=1$ iff $A=B$.

Theorem 6. Let $R$ be compatible with an $\mathbf{L}$-equivalence $\sim$ on $X$. Then $R^{+}$is compatible with the power $\mathbf{L}$-equivalence $\sim^{+}$.

Proof. By Lemma12 $\sim^{+}$is indeed an $\mathbf{L}$-equivalence. Compatibility of $R$ with $\sim$ means $\sim \circ R \circ \sim \subseteq R$. By Theorem 4, $\sim^{+} \circ R^{+} \circ \sim^{+} \subseteq(\sim \circ R \circ \sim)^{+} \subseteq R^{+}$.

The following is our main result on power relations of $\mathbf{L}$-orders.

Theorem 7. Let $\mathbf{U}=\langle\langle U, \approx\rangle, \preceq\rangle$ be an $\mathbf{L}$-ordered set, $M \subseteq L^{U}$ a subset, containing only convex $\mathbf{L}$-sets in $\mathbf{U}$. Then $\left\langle\left\langle M, \approx^{+}\right\rangle, \preceq^{+}\right\rangle$is an $\mathbf{L}$-ordered set.

Proof. Since convex $\mathbf{L}$-sets are compatible with $\approx$, then $\approx^{+}$is an $\mathbf{L}$-equality by Theorem 5 By Theorem $6 . \preceq^{+}$is compatible with $\approx^{+}$and by Lemma $12, \preceq^{+}$is reflexive and transitive.

Let $V_{1}, V_{2} \in L^{U}$ be convex. We have

$$
\begin{aligned}
& \left(V_{1} \preceq^{+} V_{2}\right) \wedge\left(V_{2} \preceq^{+} V_{1}\right)=\left(V_{1} \preceq \rightarrow V_{2}\right) \wedge\left(V_{1} \preceq \leftarrow V_{2}\right) \wedge\left(V_{2} \preceq V_{1}\right) \wedge\left(V_{2} \preceq^{\leftarrow} V_{1}\right) \\
& \quad=\left(V_{1} \preceq V_{2}\right) \wedge\left(V_{2} \preceq \rightarrow V_{1}\right) \wedge\left(V_{2} \preceq V_{1}\right) \wedge\left(V_{1} \preceq \leftarrow V_{2}\right) \\
& \quad=S\left(V_{1}, \downarrow V_{2}\right) \wedge S\left(V_{1}, \uparrow V_{2}\right) \wedge S\left(V_{2}, \downarrow V_{1}\right) \wedge S\left(V_{2}, \uparrow V_{1}\right) \\
& \quad=S\left(V_{1}, \downarrow V_{2} \cap \uparrow V_{2}\right) \wedge S\left(V_{2}, \downarrow V_{1} \cap \uparrow V_{1}\right)=S\left(V_{1}, V_{2}\right) \wedge S\left(V_{2}, V_{1}\right)=V_{1} \approx^{X} V_{2} \\
& \quad=V_{1} \approx^{+} V_{2}
\end{aligned}
$$

(the last equality follows by Lemma [13, , proving antisymmetry. 
The following two lemmas show a way of efficient computing values of power relations $\approx^{+}$and $\preceq^{+}$on intervals.

Lemma 14. Let $\mathbf{U}=\langle\langle U, \approx\rangle, \preceq\rangle$ be an $\mathbf{L}$-ordered set, $V_{1}, V_{2} \in L^{U}$ two $\mathbf{L}$-sets having minimum and maximum, $\min V_{1}=u_{1}, \max V_{1}=v_{1}, \min V_{2}=u_{2}, \max V_{2}=v_{2}$. Then $V_{1} \preceq^{+} V_{2}=\left(u_{1} \preceq u_{2}\right) \wedge\left(v_{1} \preceq v_{2}\right)$.

Proof. We will first prove that for each $w \in U$,

$$
\left(\preceq \circ V_{2}\right)(w)=w \preceq v_{2} .
$$

Since $v_{2}$ is the maximum of $V_{2}$, then by (18), Lemma 4, and (21), $V_{2} \subseteq \mathscr{L} \mathscr{U} V_{2}=$ $\mathscr{L} \mathscr{U}\left\{v_{2}\right\}=\mathscr{L}\left\{v_{2}\right\}$ whence $V_{2}\left(w^{\prime}\right) \leq \mathscr{L}\left\{v_{2}\right\}\left(w^{\prime}\right)=w^{\prime} \preceq v_{2}(20)$. Thus, for each $w \in U$,

$$
\left(w \preceq w^{\prime}\right) \otimes V_{2}\left(w^{\prime}\right) \leq\left(w \preceq w^{\prime}\right) \otimes\left(w^{\prime} \preceq v_{2}\right) \leq\left(w \preceq v_{2}\right)
$$

by transitivity. Taking supremum through all $w^{\prime}$ on the left-hand side and taking into account that $\left(w \preceq v_{2}\right) \otimes V_{2}\left(v_{2}\right)=w \preceq v_{2}$ we obtain (49).

Thus,

$$
\left(V_{1} \preceq \rightarrow V_{2}\right)=\bigwedge_{w \in U} V_{1}(w) \rightarrow\left(w \preceq v_{2}\right)=\mathscr{U} V_{1}\left(v_{2}\right)=\mathscr{U}\left\{v_{1}\right\}\left(v_{2}\right)=v_{1} \preceq v_{2}
$$

by (20).

One can prove similarly $\left(V_{1} \preceq \leftarrow V_{2}\right)=u_{1} \preceq u_{2}$ and obtain the desired equality.

Lemma 15. Let $\mathbf{U}=\langle\langle U, \approx\rangle, \preceq\rangle$ be an $\mathbf{L}$-ordered set, $V_{1}=\llbracket u_{1}, v_{1} \rrbracket, V_{2}=\llbracket u_{2}, v_{2} \rrbracket$ intervals in $U$. Then $V_{1} \approx^{+} V_{2}=\left(u_{1} \approx u_{2}\right) \wedge\left(v_{1} \approx v_{2}\right)$.

Proof. According to Theorem $7\left\langle\left\langle M, \approx^{+}\right\rangle, \preceq^{+}\right\rangle$, where $M=\left\{V_{1}, V_{2}\right\}$, is an L-ordered set. Thus by (9) and Lemma 14

$$
\begin{aligned}
V_{1} \approx^{+} V_{2} & =\left(V_{1} \preceq+V_{2}\right) \wedge\left(V_{2} \preceq^{+} V_{1}\right) \\
& =\left(u_{1} \preceq u_{2}\right) \wedge\left(v_{1} \preceq v_{2}\right) \wedge\left(u_{2} \preceq u_{1}\right) \wedge\left(v_{2} \preceq v_{1}\right) \\
& =\left(u_{1} \approx u_{2}\right) \wedge\left(v_{1} \approx v_{2}\right) .
\end{aligned}
$$

\section{Complete L-relations}

In classical theory of complete lattices (see for example [8]), a binary relation $R$ on a complete lattice $\mathbf{U}$ is called complete, if for each system $\left\{\left\langle u_{j}, v_{j}\right\rangle\right\}_{j \in J}$ of pairs of elements of $U$ from $u_{j} R v_{j}$ for each $j \in J$ it follows $\left(\bigwedge_{j \in J} u_{j}\right) R\left(\bigwedge_{j \in J} v_{j}\right)$ and $\left(\bigvee_{j \in J} u_{j}\right) R\left(\bigvee_{j \in J} v_{j}\right)$.

It can be easily checked that the following condition is equivalent to the above condition of completeness of $R$ : if $V_{1}, V_{2} \subseteq U$ are such that for each $v_{1} \in V_{1}$ there is $v_{2} \in V_{2}$ such that $v_{1} R v_{2}$ and for each $v_{2} \in V_{2}$ there is $v_{1} \in V_{1}$ such that $v_{1} R v_{2}$, then $\left(\bigwedge V_{1}\right) R\left(\bigwedge V_{2}\right)$ and $\left(\bigvee V_{1}\right) R\left(\bigvee V_{2}\right)$. 
This leads us to the following definition. A binary $\mathbf{L}$-relation on a completely lattice $\mathbf{L}$-ordered set $\mathbf{U}=\langle\langle U, \approx\rangle, \preceq\rangle$ is called complete, if it is compatible with $\approx$ and for any two $\mathbf{L}$-sets $V_{1}, V_{2} \in L^{U}$ it holds

$$
\begin{aligned}
& R^{+}\left(V_{1}, V_{2}\right) \leq R\left(\inf V_{1}, \inf V_{2}\right), \\
& R^{+}\left(V_{1}, V_{2}\right) \leq R\left(\sup V_{1}, \sup V_{2}\right) .
\end{aligned}
$$

Following are basic properties of complete relations on a completely lattice $\mathbf{L}$ ordered set $\mathbf{U}=\langle\langle U, \approx\rangle, \preceq\rangle$.

Lemma 16. If $R$ is complete, then so is $R^{-1}$.

Proof. We have

$$
\begin{aligned}
\left(R^{-1}\right)^{+}\left(V_{1}, V_{2}\right) & =\left(R^{-1}\right)^{\rightarrow}\left(V_{1}, V_{2}\right) \wedge\left(R^{-1}\right)^{\leftarrow}\left(V_{1}, V_{2}\right) \\
& =R^{\leftarrow}\left(V_{2}, V_{1}\right) \wedge R^{\rightarrow}\left(V_{2}, V_{1}\right)=R^{+}\left(V_{2}, V_{1}\right) \leq R\left(\inf V_{2}, \inf V_{1}\right) \\
& =R^{-1}\left(\inf V_{1}, \inf V_{2}\right),
\end{aligned}
$$

and similarly for suprema.

Theorem 8. The system of all complete binary $\mathbf{L}$-relations on $\mathbf{U}$ is an $\mathbf{L}$-closure system in the set $U \times U$, hence a completely lattice $\mathbf{L}$-ordered set.

Proof. We will show 1. that if $R_{j}, j \in J$, are complete then so is $\bigcap_{j \in J} R_{j}$ and 2. that for each $a \in L$ and $R$ complete the shift $a \rightarrow R$ is also complete. Since the system of all binary $\mathbf{L}$-relations that are compatible with $\approx$ is an $\mathbf{L}$-closure system, there is no need to prove compatibility of the relations.

1. We have

$$
\begin{aligned}
\left(\bigcap_{j} R_{j}\right) \circ V(v) & =\bigvee_{w \in U}\left(\bigwedge_{j} R_{j}(v, w)\right) \otimes V(w) \leq \bigwedge_{j} \bigvee_{w \in U} R_{j}(v, w) \otimes V(w) \\
& =\bigwedge_{j}\left(R_{j} \circ V\right)(v) .
\end{aligned}
$$

Thus, $\left(\bigcap_{j} R_{j}\right) \circ V \subseteq \bigcap_{j}\left(R_{j} \circ V\right)$. Now,

$$
\begin{aligned}
\left(\bigcap_{j} R_{j}\right)^{\rightarrow}\left(V_{1}, V_{2}\right) & =S\left(V_{1},\left(\bigcap_{j} R_{j}\right) \circ V_{2}\right) \leq S\left(V_{1}, \bigcap_{j}\left(R_{j} \circ V_{2}\right)\right)=\bigwedge_{j} S\left(V_{1}, R_{j} \circ V_{2}\right) \\
& =\bigwedge_{j}\left(R_{j}\right) \rightarrow\left(V_{1}, V_{2}\right)
\end{aligned}
$$


and, finally,

$$
\begin{aligned}
& \left(\bigcap_{j} R_{j}\right)^{+}\left(V_{1}, V_{2}\right)=\left(\bigcap_{j} R_{j}\right)^{\rightarrow}\left(V_{1}, V_{2}\right) \wedge\left(\bigcap_{j} R_{j}\right)^{\leftarrow}\left(V_{1}, V_{2}\right) \\
& \leq \bigwedge_{j}\left(R_{j}\right) \rightarrow\left(V_{1}, V_{2}\right) \wedge\left(R_{j}\right)^{\leftarrow}\left(V_{1}, V_{2}\right)=\bigwedge_{j}\left(R_{j}\right)^{+}\left(V_{1}, V_{2}\right) \\
& \leq \bigwedge_{j} R_{j}\left(\inf V_{1}, \inf V_{2}\right)=\left(\bigcap_{j} R_{j}\right)\left(\inf V_{1}, \inf V_{2}\right) .
\end{aligned}
$$

Similarly for suprema.

2. We have

$$
\begin{aligned}
((a \rightarrow R) \circ V)(v) & =\bigvee_{w \in U}(a \rightarrow R(v, w)) \otimes V(w) \leq a \rightarrow \bigvee_{w \in U} R(v, w) \otimes V(w) \\
& =a \rightarrow(R \circ V)(v) .
\end{aligned}
$$

Thus, $(a \rightarrow R) \circ V \subseteq a \rightarrow(R \circ V)$. Now,

$$
(a \rightarrow R)^{\rightarrow}\left(V_{1}, V_{2}\right)=S\left(V_{1},(a \rightarrow R) \circ V_{2}\right) \leq S\left(V_{1}, a \rightarrow\left(R \circ V_{2}\right)\right)=a \rightarrow S\left(V_{1}, R \circ V_{2}\right)
$$

and, finally,

$$
\begin{aligned}
(a & \rightarrow R)^{+}\left(V_{1}, V_{2}\right)=(a \rightarrow R)^{\rightarrow}\left(V_{1}, V_{2}\right) \wedge(a \rightarrow R)^{\leftarrow}\left(V_{1}, V_{2}\right) \\
& \leq\left(a \rightarrow R^{\rightarrow}\left(V_{1}, V_{2}\right)\right) \wedge\left(a \rightarrow\left(R^{\leftarrow}\left(V_{1}, V_{2}\right)\right)=a \rightarrow\left(R^{\rightarrow}\left(V_{1}, V_{2}\right) \wedge R^{\leftarrow}\left(V_{1}, V_{2}\right)\right)\right. \\
& \leq a \rightarrow R\left(\inf V_{1}, \inf V_{2}\right)=(a \rightarrow R)\left(\inf V_{1}, \inf V_{2}\right) .
\end{aligned}
$$

Similarly for suprema.

Lemma 17. The following holds for each $V_{1}, V_{2} \in L^{U}$ :

$$
V_{1} \preceq V_{2} \leq \sup V_{1} \preceq \sup V_{2}, \quad V_{1} \preceq V_{2} \leq \inf V_{1} \preceq \inf V_{2} .
$$

Proof. We have by (16), (15), (26),

$$
\begin{aligned}
V_{1} \preceq \rightarrow V_{2} & =S\left(V_{1}, \preceq \circ V_{2}\right)=S\left(V_{1}, \downarrow V_{2}\right) \leq S\left(\mathscr{U} \downarrow V_{2}, \mathscr{U} V_{1}\right)=S\left(\mathscr{U} V_{2}, \mathscr{U} V_{1}\right) \\
& =\sup V_{1} \preceq \sup V_{2} .
\end{aligned}
$$

Hence the first inequality. The second one is obtained similarly.

Theorem 9. The $\mathbf{L}$-relations $\preceq$ and $\approx$ on $\mathbf{U}$ are complete.

Proof. By Lemma[17, for each $V_{1}, V_{2} \in L^{U}, V_{1} \preceq{ }^{+} V_{2} \leq\left(V_{1} \preceq \rightarrow V_{2}\right) \leq \sup V_{1} \preceq \sup V_{2}$ and $V_{1} \preceq^{+} V_{2} \leq V_{1} \preceq \leftarrow V_{1} \leq \inf V_{1} \preceq \inf V_{2}$, proving completeness of $\preceq$.

Since $\approx=\preceq \cap \succeq$, completeness of $\approx$ follows from Lemma 16 and Theorem 8 


\section{Complete tolerances}

\subsection{Basic properties}

Recall that $\mathbf{L}$-tolerance on a set $X$ is a reflexive and symmetric binary $\mathbf{L}$-relation on $X$. For an $\mathbf{L}$-tolerance $\sim$ on a set $X$, an $\mathbf{L}$-set $B \in L^{X}$ is called a block of $\sim$ [2] if for each $x_{1}, x_{2} \in X$ it holds $B\left(x_{1}\right) \otimes B\left(x_{2}\right) \leq\left(x_{1} \sim x_{2}\right)$. A block $B$ is called maximal if for each block $B^{\prime}$ from $B \subseteq B^{\prime}$ it follows $B=B^{\prime}$. The set of all maximal blocks of $\sim$ always exists by Zorn's lemma, is called the factor set of $X$ by $\sim$ and denoted by $X / \sim$.

Further we set for each $x \in X, \llbracket x \rrbracket \sim(y)=x \sim y$, obtaining an L-set $\llbracket x \rrbracket \sim$ called the class of $\sim$ determined by $x$.

Let $\sim$ be a complete tolerance on a completely lattice $\mathbf{L}$-ordered set $\mathbf{U}=\langle\langle U, \approx$ \rangle$, \preceq\rangle$. From reflexivity of $\sim$ we have $V \subseteq \sim \circ V$ for each $V \in L^{U}$ and from symmetry $\sim^{-1}=\sim$.

For each $u \in U$ we set

$$
u_{\sim}=\inf \llbracket u \rrbracket_{\sim}, \quad u^{\sim}=\sup \llbracket u \rrbracket \sim
$$

We denote the system of all complete $\mathbf{L}$-tolerances on a completely lattice $\mathbf{L}$-ordered set $\mathbf{U}$ by CTol $\mathbf{U}$ and consider it together with the $\mathbf{L}$-equality $\approx^{U \times U}$ and $\mathbf{L}$-order $S$.

Theorem 10. CTol $\mathbf{U}$ is an $\mathbf{L}$-closure system in the set $U \times U$, hence a completely lattice L-ordered set.

Proof. Evidently, if $\sim$ is an L-tolerance then so is $a \rightarrow \sim$ for each $a \in L$ and if $\sim_{j}$, $j \in J$, are $\mathbf{L}$-tolerances then $\bigcap_{j \in J}$ is also an $\mathbf{L}$-tolerance. Thus, the theorem follows from Theorem 8

\subsection{From complete tolerances to isotone Galois connections}

Lemma 18. For each $u \in U, u \sim u_{\sim}=u \sim u^{\sim}=1$.

Proof. Set $V_{1}=\{u\}, V_{2}=\llbracket u \rrbracket \sim$. Since $V_{1} \subseteq V_{2}$, we have $V_{1} \sim V_{2}=1$. Further, $(\sim 0$ $\left.V_{1}\right)(v)=v \sim u=\llbracket u \rrbracket_{\sim}(v)$. Thus, $V_{1} \sim \leftarrow V_{2}=S\left(\llbracket u \rrbracket_{\sim}, \llbracket u \rrbracket_{\sim}\right)=1$. Now,

$$
V_{1} \sim^{+} V_{2}=\left(V_{1} \sim^{\rightarrow} V_{2}\right) \wedge\left(V_{1} \sim^{\leftarrow} V_{2}\right)=1
$$

and by completeness of $\sim, 1=\inf V_{1} \sim \inf V_{2}=u \sim u \sim$ and $1=\sup V_{1} \sim \sup V_{2}=u \sim$ $u^{\sim}$.

Lemma 19. It holds for any $u \in U$

$$
u_{\sim}^{\sim} \geq u, \quad u^{\sim} \sim \leq u
$$

Proof. By Lemma 18 $\llbracket u \rrbracket \sim\left(u_{\sim}\right)=1$. This means that also $\llbracket u_{\sim} \rrbracket \sim(u)=1$. Since $u_{\sim}^{\sim}=$ $\sup \llbracket u \sim \rrbracket \sim$, we have the first inequality.

The second inequality is analogous. 
Lemma 20. For each $u, v \in U$ it holds

$$
(u \preceq v) \leq\left(u \sim \preceq v_{\sim}\right), \quad(u \preceq v) \leq\left(u^{\sim} \preceq v^{\sim}\right) .
$$

Proof. Let $a=u \preceq v, V_{1}=\{a / u, v\}, V_{2}=\left\{a / u^{\sim}, v^{\sim}\right\}$. By Lemma 18, $u \sim u^{\sim}=v \sim$ $v^{\sim}=1$. Thus, $V_{1} \subseteq \sim \circ V_{2}, V_{2} \subseteq \sim \circ V_{1}$ and we have $V_{1} \sim^{+} V_{2}=1$. By completeness of $\sim, \sup V_{1} \sim \sup V_{2}=1$.

By Lemma $6 \sup V_{1}=v$. Thus, $v \sim \sup V_{2}=1$, which means $\sup V_{2} \leq v^{\sim}$. On the other hand, since $V_{2}\left(v^{\sim}\right)=1$, we have $\sup V_{2} \geq v^{\sim}$, whence $\sup V_{2}=v^{\sim}$. By Lemma4, $V_{2} \subseteq \mathscr{L}\left\{v^{\sim}\right\}$. Thus, $a=V_{2}\left(u^{\sim}\right) \leq \mathscr{L}\left\{v^{\sim}\right\}\left(u^{\sim}\right)=u^{\sim} \preceq v^{\sim}$ and the second inequality in (54) is proved.

The first inequality is proved similarly.

Theorem 11. The pair $\left\langle\sim,^{\sim}\right\rangle$ is an extensive isotone Galois connection on $\mathbf{U}$.

Proof. We will show $\langle\sim, \sim\rangle$ is an isotone Galois connection. Let $u, v \in U$. We have by Lemma 20, Lemma 19 , and transitivity of $\preceq$,

$$
(u \sim \preceq v) \leq\left(u_{\sim}^{\sim} \preceq v^{\sim}\right) \leq\left(u \preceq v^{\sim}\right) .
$$

The converse inequality is proven analogously.

Extensivity of $\langle\sim, \sim\rangle$ follows trivially from reflexivity of $\preceq$.

\subsection{Structure of maximal blocks}

Lemma 21. If $\langle u, v\rangle$ is a fixpoint of $\left\langle\sim,^{\sim}\right\rangle$, then $\llbracket v, u \rrbracket$ is a block of $\sim$.

Proof. 1. We will prove that for each $w$,

$$
\llbracket v, u \rrbracket(w) \leq u \sim w
$$

(i.e. "if $w$ belongs to $\llbracket v, u \rrbracket$, then it is similar to $u$ ").

Set $a=w \preceq u, b=v \preceq w, V_{1}=\left\{u,{ }^{a} / w\right\}, V_{2}=\{b / v, w\}$. By Lemma $6, \sup V_{1}=u$ and $\sup V_{2}=w$.

Now,

$$
\begin{aligned}
& \left(\sim \circ V_{1}\right)(v)=\left((v \sim u) \otimes V_{1}(u)\right) \vee\left((v \sim w) \otimes V_{1}(w)\right)=(1 \otimes 1) \vee((v \sim w) \otimes a)=1, \\
& \left(\sim \circ V_{1}\right)(w)=\left((w \sim u) \otimes V_{1}(u)\right) \vee\left((w \sim w) \otimes V_{1}(w)\right)=((w \sim u) \otimes 1) \vee(1 \otimes a) \geq a, \\
& \left(\sim \circ V_{2}\right)(u)=\left((u \sim v) \otimes V_{2}(v)\right) \vee\left((u \sim w) \otimes V_{2}(w)\right)=(1 \otimes b) \vee((u \sim w) \otimes 1) \geq b, \\
& \left(\sim \circ V_{2}\right)(w)=\left((w \sim v) \otimes V_{2}(v)\right) \vee\left((w \sim w) \otimes V_{2}(w)\right)=((w \sim v) \otimes b) \vee(1 \otimes 1)=1
\end{aligned}
$$

Thus,

$$
\begin{aligned}
& S\left(V_{1}, \sim \circ V_{2}\right)=\left(V_{1}(u) \rightarrow\left(\sim \circ V_{2}\right)(u)\right) \wedge\left(V_{1}(w) \rightarrow\left(\sim \circ V_{2}\right)(w)\right) \geq b, \\
& S\left(V_{2}, \sim \circ V_{1}\right)=\left(V_{2}(v) \rightarrow\left(\sim \circ V_{1}\right)(v)\right) \wedge\left(V_{2}(w) \rightarrow\left(\sim \circ V_{1}\right)(w)\right) \geq a
\end{aligned}
$$


and

$$
\begin{aligned}
\llbracket v, u \rrbracket(w) & =a \wedge b \leq S\left(V_{1}, \sim \circ V_{2}\right) \wedge S\left(V_{2}, \sim \circ V_{1}\right)=V_{1} \sim^{+} V_{2} \\
& \leq \sup V_{1} \sim \sup V_{2}=u \sim w,
\end{aligned}
$$

proving (55).

2. Let $w_{1}, w_{2} \in U, a_{1}=\llbracket v, u \rrbracket\left(w_{1}\right), a_{2}=\llbracket v, u \rrbracket\left(w_{2}\right), b_{1}=w_{1} \preceq u, b_{2}=w_{2} \preceq u$. By (55), $a_{1} \leq b_{1}, a_{2} \leq b_{2}$. Set $V_{1}=\left\{b_{1} / u, w_{1}\right\}, V_{2}=\left\{b_{2} / u, w_{2}\right\}$. By similar direct calculations as above we obtain

$$
a_{1} \otimes a_{2} \leq a_{1} \otimes b_{2}=\left(b_{1} \rightarrow b_{2}\right) \wedge\left(1 \rightarrow b_{2} \otimes a_{1}\right) \leq V_{1} \sim \rightarrow V_{2} .
$$

Similarly, $a_{1} \otimes a_{2} \leq V_{1} \sim \leftarrow V_{2}$ and

$$
a_{1} \otimes a_{2} \leq V_{1} \sim^{+} V_{2} \leq \inf V_{1} \sim \inf V_{2}=w_{1} \sim w_{2},
$$

proving $\llbracket v, u \rrbracket$ is a block.

Lemma 22. If $B$ is a block of $\sim$, then so is $B \cup\{\inf B\}$.

Proof. Let $u=\inf B$. It suffices to prove $B(v) \leq u \sim v$ for each $v \in U$.

Let $V=\{v\}$. We have

$$
\begin{aligned}
& B \sim \rightarrow V=\bigwedge_{w \in U} B(w) \rightarrow(v \sim w) \geq B(v), \\
& B \sim^{\leftarrow} V=B(v) .
\end{aligned}
$$

Thus,

$$
B(v) \leq B \sim^{+} V \leq \inf B \sim \inf V=u \sim v
$$

and the lemma is proved.

Lemma 23. For each block $B$ of $\sim$ there is a fixpoint $\langle u, v\rangle$ of $\left\langle\sim,^{\sim}\right\rangle$ such that $B \subseteq \llbracket v, u \rrbracket$.

Proof. Set $w=\inf B, u=w^{\sim}, v=u_{\sim}$. Since $\langle\sim, \sim\rangle$ is an isotone Galois connection (Theorem 11), $\langle u, v\rangle$ is a fixpoint. By Lemma22, the $\mathbf{L}$-set $B^{\prime}=B \cup\{w\}$ is again a block. By Lemma 4, we have $B^{\prime} \subseteq \mathscr{U}\{w\} \subseteq \mathscr{U}\{v\}$. Also, for each $w^{\prime}$ it holds $B^{\prime}\left(w^{\prime}\right) \leq w^{\prime} \sim w$. Thus, by definition of class, $B^{\prime} \subseteq \llbracket w \rrbracket_{\sim}$, whence $\sup B^{\prime} \leq \sup \llbracket w \rrbracket \sim=u$ (27). This yields $B^{\prime} \subseteq \mathscr{L}\{u\}$ and we can conclude $B \subseteq B^{\prime} \subseteq \mathscr{U}\{v\} \cap \mathscr{L}\{u\}=\llbracket v, u \rrbracket$.

Theorem 12. Maximal blocks of $\sim$ are exactly intervals $\llbracket v, u \rrbracket$, where $\langle u, v\rangle$ are fixpoints of $\langle\sim, \sim\rangle$.

Proof. Follows from the above lemmas. 


\subsection{Structure of classes}

Theorem 13. For each $u \in U$, the class $\llbracket u \rrbracket \sim$ is equal to the interval $\llbracket u_{\sim}, u^{\sim} \rrbracket$.

Proof. By Lemma $4 \llbracket \llbracket u \rrbracket_{\sim} \subseteq \mathscr{U}\left\{\inf \llbracket u \rrbracket_{\sim}\right\}=\mathscr{U}\left\{u_{\sim}\right\}$ and similarly $\llbracket u \rrbracket_{\sim} \subseteq \mathscr{L}\left\{u^{\sim}\right\}$. Thus, $\llbracket u \rrbracket \sim \subseteq \llbracket u_{\sim}, u^{\sim} \rrbracket$.

Let $u^{\prime} \in U, a=\llbracket u_{\sim}, u^{\sim} \rrbracket\left(u^{\prime}\right)=\left(u_{\sim} \preceq u^{\prime}\right) \wedge\left(u^{\prime} \preceq u^{\sim}\right)$. We will show the $\mathbf{L}$-set $V=$ $\left\{a / u^{\prime}, u\right\}$ is a block. For the lower cone of $V$ we have

$$
\mathscr{L} V(w)=(w \preceq u) \wedge\left(a \rightarrow\left(w \preceq u^{\prime}\right)\right) .
$$

Let $v=\inf V$. According to Lemma $4, V \subseteq \mathscr{U}\{v\}$. By Lemma 19 $v^{\sim} \sim \leq v$, whence $V \subseteq \mathscr{U}\left\{v^{\sim} \sim\right\}$.

Now consider membership degrees of $u$ and $u^{\prime}$ in the lower cone $\mathscr{L}\left\{v^{\sim}\right\}$. Since $a \leq u_{\sim} \preceq u^{\prime}$, then (56) $\mathscr{L} V\left(u_{\sim}\right)=1 \wedge\left(a \rightarrow\left(u_{\sim} \preceq u^{\prime}\right)\right)=1$. Thus, $1=u \sim \preceq v=u \preceq$ $v^{\sim}=\mathscr{L}\left\{v^{\sim}\right\}(u)$, obtaining $\mathscr{L}\left\{v^{\sim}\right\}(u)=1$.

For $\mathscr{L}\left\{v^{\sim}\right\}\left(u^{\prime}\right)$ we first notice by (56), $\mathscr{L} V\left(u_{\sim}^{\prime}\right)=u_{\sim}^{\prime} \preceq u=u^{\prime} \preceq u^{\sim} \geq a$. By Lemma 4, and (20), $\mathscr{L} V\left(u_{\sim}^{\prime}\right)=\mathscr{L}\{v\}\left(u_{\sim}^{\prime}\right)=u_{\sim}^{\prime} \preceq v$, and $\mathscr{L}\left\{v^{\sim}\right\}\left(u^{\prime}\right)=u^{\prime} \preceq v^{\sim}=$ $u_{\sim}^{\prime} \preceq v \geq a$. Thus, $V \subseteq \mathscr{L}\left\{v^{\sim}\right\}$.

Together, $V \subseteq \mathscr{U}\left\{v^{\sim} \sim\right\} \cap \mathscr{L}\left\{v^{\sim}\right\}=\llbracket v^{\sim} \sim, v^{\sim} \rrbracket$. By Theorem 12 $\llbracket v^{\sim}, v^{\sim} \rrbracket$ is a block. Thus, $V$ is also a block and by definition of block we obtain $\llbracket u_{\sim}, u^{\sim} \rrbracket\left(u^{\prime}\right)=$ $V\left(u^{\prime}\right)=V\left(u^{\prime}\right) \otimes V(u) \leq u^{\prime} \sim u=\llbracket u \rrbracket \sim\left(u^{\prime}\right)$. Thus, $\llbracket u_{\sim}, u^{\sim} \rrbracket \subseteq \llbracket u \sim_{\sim}$ and the theorem is proved.

The following is an important consequence of Theorem 13 that we will use later to prove our main result.

Lemma 24. For each $u, v \in U$ we have

$$
u \sim v=(u \sim \preceq v) \wedge\left(v \preceq u^{\sim}\right) .
$$

Proof. The right-hand side is equal to $\llbracket u_{\sim}, u^{\sim} \rrbracket(v)$, which is by Theorem 13 equal to $\llbracket u \rrbracket \sim(v)=u \sim v$.

We use the above results in the proof of the following lemma. By Theorem 11 for each complete $\mathbf{L}$-tolerance $\sim$ on $\mathbf{U}$ the pair $\langle\sim, \sim\rangle$ is an isotone $\mathbf{L}$-Galois connection. Thus, we can $\mathbf{L}$-order such $\mathbf{L}$-Galois connections by the $\mathbf{L}$-relation $\preceq_{\mathrm{IGal}(\mathbf{U}, \mathbf{U})}$ (37).

Lemma 25. For any two complete $\mathbf{L}$-tolerances $\sim_{1}, \sim_{2}$ on $\mathbf{U}$ we have

$$
S\left(\sim_{1}, \sim_{2}\right)=\left\langle\sim_{1}, \sim_{1}\right\rangle \preceq_{\mathrm{IGal}(\mathbf{U}, \mathbf{U})}\left\langle\sim_{\sim_{2}}, \sim_{2}\right\rangle .
$$

Proof. By definitions of $S$ and $\preceq_{\operatorname{IGal}(\mathbf{U}, \mathbf{U})}$ we have to prove the following equality:

$$
\bigwedge_{u, v \in U}\left(u \sim_{1} v\right) \rightarrow\left(u \sim_{2} v\right)=\bigwedge_{u \in U}\left(u_{\sim_{2}} \preceq u_{\sim_{1}}\right) \wedge \bigwedge_{u \in U}\left(u^{\sim_{1}} \preceq u^{\sim_{2}}\right) .
$$

We will proceed by proving both inequalities " $\leq$ " and " $\geq$ ". 
" $\leq$ ": Since $u \sim_{1} u^{\sim 1}$ (Lemma 18), the left-hand side of (58) is $\leq \bigwedge_{u \in U} u \sim_{2} u^{\sim 1}$. Now by Theorem 13 and (20) we have

$$
\begin{aligned}
u \sim_{2} u^{\sim_{1}} & =\llbracket u^{\sim_{1}} \rrbracket_{\sim_{2}}(u)=\mathscr{L}\left\{\left(u^{\sim_{1}}\right)^{\sim_{2}}\right\}(u) \wedge \mathscr{U}\left\{\left(u^{\sim_{1}}\right)_{\sim_{2}}\right\}(u) \\
& \leq \mathscr{U}\left\{\left(u^{\sim 1}\right)_{\sim_{2}}\right\}(u)=\left(u^{\sim_{1}}\right)_{\sim_{2}} \preceq u=u^{\sim 1} \preceq u^{\sim_{2}} .
\end{aligned}
$$

Thus, $\bigwedge_{u, v \in U}\left(u \sim_{1} v\right) \rightarrow\left(u \sim_{2} v\right) \leq \bigwedge_{u \in U} u^{\sim_{1}} \preceq u^{\sim_{2}}$. The inequality $\bigwedge_{u, v \in U}\left(u \sim_{1} v\right) \rightarrow$ $\left(u \sim_{2} v\right) \leq \bigwedge_{u \in U} u_{\sim_{2}} \preceq u_{\sim_{1}}$ is proved similarly.

" $\geq$ ": by Theorem 13 and (20) again and by antisymmetry of $\preceq$ we obtain

$$
\begin{aligned}
& \left(u^{\sim_{1}} \preceq u^{\sim_{2}}\right) \otimes\left(u \sim_{1} v\right) \leq\left(u^{\sim_{1}} \preceq u^{\sim_{2}}\right) \otimes\left(\left(v \preceq u^{\sim_{1}}\right) \wedge\left(u_{\sim_{1}} \preceq v\right)\right) \\
& \quad \leq\left(u^{\sim_{1}} \preceq u^{\sim^{2}}\right) \otimes\left(v \preceq u^{\sim_{1}}\right) \leq v \preceq u^{\sim^{2}} .
\end{aligned}
$$

Similarly $\left(u_{\sim_{2}} \preceq u_{\sim_{1}}\right) \otimes\left(u \sim_{1} v\right) \leq\left(u_{\sim_{2}} \preceq v\right)$, thereby (Theorem[13 and (20)

$$
\begin{aligned}
\left(u \sim_{2} v\right) & =\left(u \sim_{2} \preceq v\right) \wedge\left(v \preceq u^{\sim_{2}}\right) \\
& \geq\left(\left(u^{\sim 1} \preceq u^{\sim 2}\right) \otimes\left(u \sim_{1} v\right)\right) \wedge\left(\left(u \sim_{2} \preceq u \sim_{1}\right) \otimes\left(u \sim_{1} v\right)\right) \\
& \geq\left(\left(u^{\sim_{1}} \preceq u^{\sim_{2}}\right) \wedge\left(u_{\sim_{2}} \preceq u u_{\sim_{1}}\right)\right) \otimes\left(u \sim_{1} v\right) .
\end{aligned}
$$

By adjointness,

$$
\left(u \sim_{1} v\right) \rightarrow\left(u \sim_{2} v\right) \geq\left(u^{\sim_{1}} \preceq u^{\sim_{2}}\right) \wedge\left(u \sim_{2} \preceq u_{\sim_{1}}\right),
$$

yielding the " $\geq$ " part of $(58$.

\subsection{From extensive isotone Galois connections to complete tolerances}

Let $\langle f, g\rangle$ be an extensive isotone $\mathbf{L}$-Galois connection on a completely lattice $\mathbf{L}$-ordered set $\mathbf{U}=\langle\langle U, \approx\rangle, \preceq\rangle$. We set for each $u, v \in U$,

$$
u \sim_{\langle f, g\rangle} v=(f(u) \preceq v) \wedge(v \preceq g(u)) .
$$

The following theorem summarizes main properties of the $\mathbf{L}$-relation $\sim_{\langle f, g\rangle}$.

Theorem 14. $\sim_{\langle f, g\rangle}$ is a complete tolerance such that for each $u \in U$,

$$
u_{\sim\langle f, g\rangle}=f(u), \quad u^{\sim\langle f, g\rangle}=g(u) .
$$

Proof. The $\mathbf{L}$-relation $\sim_{\langle f, g\rangle}$ is evidently reflexive and symmetric, hence an $\mathbf{L}$-tolerance.

Set $R(u, v)=u \preceq g(v)$. We have $u \sim_{\langle f, g\rangle} v=R(u, v) \wedge R^{-1}(u, v)$. Thus, by Lemma16 and Theorem 8 it is sufficient to prove that $R$ is complete.

Let $V \in L^{U}$. Using obvious inequality $V(w) \leq g(V)(g(w))$ we have

$$
\begin{aligned}
(R \circ V)(v) & =\bigvee_{w \in U} R(v, w) \otimes V(w)=\bigvee_{w \in U}(v \preceq g(w)) \otimes V(w) \\
& \leq \bigvee_{w \in U}(v \preceq g(w)) \otimes g(V)(g(w)) \leq \bigvee_{w^{\prime} \in U}\left(v \preceq w^{\prime}\right) \otimes g(V)\left(w^{\prime}\right) \\
& =(\preceq \circ g(V))(v)
\end{aligned}
$$


and

$$
\begin{aligned}
\left(R^{-1} \circ V\right)(v) & =\bigvee_{w \in U} R(w, v) \otimes V(w)=\bigvee_{w \in U}(v \succeq f(w)) \otimes V(w) \\
& \leq \bigvee_{w \in U}(v \succeq f(w)) \otimes f(V)(f(w)) \leq \bigvee_{w^{\prime} \in U}(v \succeq f(w)) \otimes f(V)\left(w^{\prime}\right) \\
& =(\succeq \circ f(V))(v),
\end{aligned}
$$

whence $R^{\rightarrow}\left(V_{1}, V_{2}\right)=S\left(V_{1}, R \circ V_{2}\right) \leq S\left(V_{1}, \preceq \circ g\left(V_{2}\right)\right)=V_{1} \preceq \rightarrow g\left(V_{2}\right)$ and $R^{\leftarrow}\left(V_{1}, V_{2}\right)=$ $S\left(V_{2}, R^{-1} \circ V_{1}\right) \leq S\left(V_{2}, \succeq \circ f\left(V_{1}\right)\right)=f\left(V_{1}\right) \preceq \leftarrow V_{2}$.

Now by Lemma 17 and Theorem 1 (d),

$$
\begin{aligned}
R^{+}\left(V_{1}, V_{2}\right) & \leq R^{\rightarrow}\left(V_{1}, V_{2}\right) \leq V_{1} \preceq g\left(V_{2}\right) \leq \sup V_{1} \preceq \sup g\left(V_{2}\right) \\
& \leq \sup V_{1} \preceq g\left(\sup V_{2}\right)=R\left(\sup V_{1}, \sup V_{2}\right), \\
R^{+}\left(V_{1}, V_{2}\right) & \leq R^{\leftarrow}\left(V_{1}, V_{2}\right) \leq f\left(V_{1}\right) \preceq \leftarrow V_{2} \leq \inf f\left(V_{1}\right) \preceq \inf V_{2} \\
& \leq f\left(\inf V_{1}\right) \preceq \inf V_{2}=R\left(\inf V_{1}, \inf V_{2}\right),
\end{aligned}
$$

proving completeness of $R$ and hence of $\sim\langle f, g\rangle$.

To prove (60), we notice that for each $u \in U$ the class $\llbracket u \rrbracket_{\sim_{\langle f, g\rangle}}$ is equal to the interval $\llbracket f(u), g(u) \rrbracket:$

$$
\begin{aligned}
\llbracket u \rrbracket_{\sim_{\langle f, g\rangle}}(v) & =u \sim_{\langle f, g\rangle} v=(f(u) \preceq v) \wedge(v \preceq g(u)) \\
& =\mathscr{U}\{f(u)\}(v) \wedge \mathscr{L}\{g(u)\}(v)=\llbracket f(u), g(u) \rrbracket(v) .
\end{aligned}
$$

Now, $u_{\sim\langle f, g\rangle}=\inf \llbracket f(u), g(u) \rrbracket=f(u)$ and $u^{\sim\langle f, g\rangle}=\sup \llbracket f(u), g(u) \rrbracket=g(u)$.

\subsection{Factorization theorem, representation theorem}

By Theorem 12, the factor set $U / \sim$ consists of intervals. Thus, by Theorem 7 , the tuple $\mathbf{U} / \sim=\left\langle\left\langle U / \sim, \widetilde{\sim}^{+}\right\rangle, \preceq^{+}\right\rangle$is an $\mathbf{L}$-ordered set. By Theorem 11, the pair $\left\langle\sim,{ }^{\sim}\right\rangle$ is an extensive isotone Galois connection. The following theorem connects $\mathbf{U} / \sim$ to the completely lattice L-ordered set Fix $\langle\sim, \sim\rangle$.

Theorem 15 (factorization theorem). The $\mathbf{L}$-ordered set $\mathbf{U} / \sim$ is isomorphic to the completely lattice $\mathbf{L}$-ordered set $\left.\mathrm{Fix}_{\langle\sim, \sim}\right\rangle$ and, as such, is itself a completely lattice $\mathbf{L}$ ordered set. The isomorphism is given by $\llbracket v, u \rrbracket \rightarrow\langle u, v\rangle$.

Proof. Follows directly from Lemma 15, 14 and definition of $\mathbf{L}$-order on Fix $\langle\sim, \sim\rangle$.

The second main result is that complete tolerances on completely lattice $\mathbf{L}$-ordered sets can be represented by extensive isotone Galois connections.

Theorem 16 (representation theorem). The mapping

$$
\sim \mapsto\langle\sim, \sim\rangle
$$

is an isomorphism between $\mathrm{CTol} \mathbf{U}$ and $\mathrm{EIGal}(\mathbf{U})$. Its inverse is

$$
\langle f, g\rangle \mapsto \sim\langle f, g\rangle .
$$

$\mathrm{CTol} \mathbf{U}$ and $\mathrm{EIGal}(\mathbf{U})$ are both completely lattice $\mathbf{L}$-ordered sets.

Proof. Follows from Theorem 11, Lemma 24, Theorem 14, Lemma 25, and Theorem 10. 


\section{Conclusion}

We introduced a notion of complete binary fuzzy relation on complete fuzzy lattice (completely lattice fuzzy ordered set). The notion leads in ordinary (crisp) case to the classical notion of complete relation on complete lattice, but re-formulated in terms of the theory of power structures. We proved some basic properties of power structures of fuzzy ordered sets.

In the main part of the paper, we defined complete fuzzy binary relations and complete fuzzy tolerances and investigated their properties. Our main results are covered in Theorem 15 and 16. We show that a fuzzy complete lattice can be factorized by means of a complete fuzzy tolerance and that there is a naturally-defined structure of fuzzy complete lattice on the factor set. This result corresponds to the known result from the ordinary case [7|16].

In addition, we found an isomorphism between the fuzzy ordered sets of all complete fuzzy tolerances and extensive isotone fuzzy Galois connections on a fuzzy complete lattice. This result is useful for testing fuzzy tolerances for completeness and is new even in the ordinary (crisp) setting.

Our future research will focus on applying results from this paper to Formal Concept Analysis of data with fuzzy attributes [2]. In ordinary setting, there is a correspondence between complete tolerances on a concept lattice and so called block relations of the associated formal context [16]8]. Theorem 15$]$ and 16 will help establish a link between complete fuzzy tolerances on a fuzzy concept lattice and (properly defined) block relations on the formal context. This will allow generalize results from [16]8] to fuzzy concept lattices.

One of the consequences of our results is that the condition of compatibility from the definition of complete relation on a completely lattice $\mathbf{L}$-ordered set (Sec. (4) is re-

dundant for $\mathbf{L}$-tolerances. This leads to an open problem, namely, whether the condition of compatibility follows from the other conditions of the definition.

\section{References}

1. R. Belohlavek, Concept lattices and order in fuzzy logic, Ann. Pure Appl. Log. 128 (1-3) (2004) 277-298.

2. R. Belohlavek, Fuzzy Relational Systems: Foundations and Principles, Kluwer Academic Publishers, Norwell, USA, 2002.

3. R. Belohlavek, Lattice type fuzzy order and closure operators in fuzzy ordered sets, in: IFSA World Congress and 20th NAFIPS International Conference, 2001. Joint 9th, Vol. 4, 2001, pp. 2281-2286 vol.4.

4. R. Belohlavek, J. Dvorak, J. Outrata, Fast factorization by similarity in formal concept analysis of data with fuzzy attributes, Journal of Computer and System Sciences 73 (6) (2007) 1012-1022.

5. I. Bošnjak, R. Madarász, On the composition of fuzzy power relations, Fuzzy Sets and Systems,In press.

6. C. Brink, Power structures, Algebra universalis 30 (2) (1993) 177-216.

7. G. Czédli, Factor lattices by tolerances, Acta Sci. Math. 44 (1982) 35-42.

8. B. Ganter, R. Wille, Formal Concept Analysis - Mathematical Foundations, Springer, 1999. 
9. G. Georgescu, Fuzzy power structures, Archive for Mathematical Logic 47 (3) (2008) 233261.

10. G. Georgescu, A. Popescu, Non-dual fuzzy connections, Arch. Math. Log. 43 (8) (2004) 1009-1039.

11. P. Hájek, Metamathematics of Fuzzy Logic (Trends in Logic), Springer, 2001.

12. W. Kohout, L. J.; Bandler, Relational-product architectures for information processing, Information Sciences 37 (1-3) (1985) 25-37.

13. J. Konecny, Isotone fuzzy galois connections with hedges, Inf. Sci. 181 (10) (2011) 18041817.

14. M. Krupka, Factorization of residuated lattices, Log. J. IGPL 17 (2) (2009) 205-223.

15. M. Ward, R. P. Dilworth, Residuated lattices, Transactions of the American Mathematical Society 45 (1939) 335-354.

16. R. Wille, Complete tolerance relations of concept lattices, in: G. Eigenthaler, et al. (Eds.), Contributions to General Algebra, Vol. 3, Hölder-Pichler-Tempsky, Wien, 1985, pp. 397415.

17. L. A. Zadeh, The concept of a linguistic variable and its application to approximate reasoning, part I, Journal of Information Science (1975) 199-249. 